\title{
Colonic Mucosal Microbiota in Colorectal Cancer: A Single-Center Metagenomic Study in Saudi Arabia
}

\author{
Ahmed O. Alomair $\mathbb{D}^{1}{ }^{1}$ Ibrahim Masoodi $\mathbb{D}^{1,2}{ }^{1,2}$ Essam J. Alyamani, ${ }^{3}$ Abed A. Allehibi, ${ }^{1}$ \\ Adel N. Qutub, ${ }^{1}$ Khalid N. Alsayari, ${ }^{1}$ Musaad A. Altammami, ${ }^{3}$ and Ali S. Alshanqeeti ${ }^{3,4}$ \\ ${ }^{1}$ Gastroenterology \& Hepatology Department, King Fahad Medical City, Riyadh, Saudi Arabia \\ ${ }^{2}$ Department of Medicine, College of Medicine, University of Taif, Taif, Saudi Arabia \\ ${ }^{3}$ National Center for Biotechnology, King Abdulaziz City for Science and Technology (KACST), Riyadh, Saudi Arabia \\ ${ }^{4}$ National Blood \& Cancer Center, Riyadh, Saudi Arabia
}

Correspondence should be addressed to Ahmed O. Alomair; alomair21@yahoo.com

Received 5 December 2017; Revised 22 March 2018; Accepted 10 April 2018; Published 16 May 2018

Academic Editor: Matteo Frasson

Copyright (c) 2018 Ahmed O. Alomair et al. This is an open access article distributed under the Creative Commons Attribution License, which permits unrestricted use, distribution, and reproduction in any medium, provided the original work is properly cited.

Background and Aim. Because genetic and geographic variations in intestinal microbiota are known to exist, the focus of this study was to establish an estimation of microbiota in colorectal cancer (CRC) patients in Saudi Arabia by means of metagenomic studies. Methods. From July 2010 to November 2012, colorectal cancer patients attending our hospital were enrolled for the metagenomic studies. All underwent clinical, endoscopic, and histological assessment. Mucosal microbiota samples were collected from each patient by jet-flushing colonic mucosa with distilled water at unified segments of the colon, followed by aspiration, during colonoscopy. Total purified dsDNA was extracted and quantified prior to metagenomic sequencing using an Illumina platform. Satisfactory DNA samples $(n=29)$ were subjected to metagenomics studies, followed by comprehensive comparative phylogenetic analysis. An equal number of healthy age-matched controls were also examined for colonic mucosal microbiota. Results. Metagenomics data on 29 patients (14 females) in the age range 38-77 years were analyzed. The majority 11 (37\%) of our patients were overweight $(\mathrm{BMI}=25-30)$. Rectal bleeding was the presenting symptom in $18 / 29(62 \%)$, while symptomatic anemia was the presenting symptom in 11/29 (37\%). The location of colon cancer was rectal in 14 (48\%), while cecal growth was observed in 8 (27\%). Hepatic flexure growth was found in $1(3 \%)$, descending colonic growth was found in 2 (6\%), and 4 (13\%) patients had transverse colon growth. The metagenomics analysis was carried out, and a total of $3.58 \mathrm{G}$ reads were sequenced, and about $321.91 \mathrm{G}$ data were used in the analysis. This study identified 11 genera specific to colorectal cancer patients when compared to genera in the control group. Bacteroides fragilis and Fusobacterium were found to be significantly prevalent in the carcinoma group when compared to the control group. Conclusion. The current study has given an insight into the microbiota of colorectal cancer patients in Saudi Arabia and has identified various genera significantly present in these patients when compared to those of the control group.

\section{Introduction}

Metagenomics is a molecular method of culture-independent microbiology, in which genetic material recovered directly from environmental samples is studied. It has emerged as one of the most robust sequence-driven approaches for studying the composition and the genetic potential of the mucosal gut microbiota. Metagenomics analysis has begun to demonstrate the breadth of the functional and metabolic potential of microbes. It has been used to demonstrate significant metabolic discrepancies between diseased and healthy individuals. Although the intestinal microbiota in individuals reflects great variations among people according to their age, geographic origin, state of health, and variations in diet, it tends to remain stable over long periods [1].

An alteration in gut microbiota can cause the development of inflammation within the colon, and such inflammation is implicated in colonic neoplastic development. Although the precise mechanisms through which the microbiota is involved in cancer development remain elusive, 
the message is, however, clear: the microbiota contributes to cancer risk by influencing some fundamental host processes [2]. This implies that modifiable risk factor interventions to modulate gut microbiota can contribute to decreasing the morbidity and mortality rates in colon cancer.

The risk of developing colorectal cancer (CRC) varies markedly between and within populations and geographical regions [3]. Accordingly, various aspects of carcinogenesis in colon cancer have been addressed in Saudi Arabia by different investigators from time to time [4-8], but to the best of our knowledge and after a literature search, there appears to be no existing data regarding the role of microbiota in CRC in this part of the globe. Hence, we were prompted to undertake this study, first of its kind in Saudi Arabia, in which we have tried to introspect the role of mucosal intestinal microbiota in CRC patients.

It will be prudent to mention that the mucosal microbiota lives closer to the intestinal epithelium when compared to the luminal microbiota, and conceivably, it would be interacting more directly with the host immune system than would the luminal/fecal bacteria. It is quite possible that mucosal microbiota might be more directly involved in inducing colon carcinogenesis. In addition, the availability of nutrients in the mucus layer of the epithelium is also entirely different from that in the gut lumen environment. Substantial differences in mucosal and fecal microbial composition have been shown to exist $[9,10]$. Hence, we choose mucosal samples and not fecal samples for this metagenomic study in CRC patients. The microbiota was compared with that in ageand gender-matched controls.

\section{Material and Methods}

This study was conducted in full compliance with the guidelines for good clinical practice of the World Medical Assembly Declaration of Helsinki and the research guidelines of the King Fahad Medical City (KFMC), Riyadh. The study was approved both by the Ethics Committee of King Abdulaziz City for Science and Technology and KFMC, Riyadh.

2.1. Inclusion Criteria for Study Participants. During the study period (July 2010 and November 2012), all colonoscopy patients at King Fahad Medical City (KFMC), Riyadh, were asked to provide specific written consent for possible inclusion in this study. Samples collected during the colonoscopy procedures in the following types of patient were then processed.

(1) Cases

(a) Adult patients with diagnosed colorectal cancer whose diagnoses were based on endoscopic and histological criteria.

(2) Controls

(a) Patients undergoing screening colonoscopic examination with normal colonoscopic procedures. (b) Patients undergoing colonoscopic procedures for lower GI bleed who had been proved to have hemorroids and anal fissures.

(c) Patients with abdominal pain undergoing the colonoscopic procedure and who proved to have a normal colonoscopic examination

2.2. Exclusion Criteria. Patients with obstruction at presentation due to colon cancer were excluded. Patients who had used antibiotics two weeks prior to colonoscopy were excluded.

2.3. Data Collection. Demographic and clinical data were collected from all participants, including age, sex, and body mass index (BMI). Detailed medical histories, including diet, comorbidities, and the chronological order of any medications taken or procedures performed, were also obtained from each participant, and the data was entered in a Microsoft Excel file.

2.4. Bowel Preparation and Sample Collection. After a standard bowel preparation that included polyethylene glycol colonic preparation, a full-length colonoscopy was carried out. During the procedure, distilled water was pushed through the biopsy channel of the scope which was collected back by aspiration. The mucosal jet wash from unified segments of the colon (cecum, transverse, left, and rectal colon segments) was collected from all study participants. The healthy mucosa of each study participant was also sampled. Sites of macroscopic mucosal abnormality in any of the four selected segments were also included. Finally, $50 \mathrm{~mL}$ of the washes, along with the remaining colonic fluids, was aspirated through the working suction and biopsy channel. All segment samples obtained from each colonoscopy were collected in four $15 \mathrm{~mL}$ test tubes and immediately stored at $-80^{\circ} \mathrm{C}$ for further processing.

2.5. DNA Extraction for Total Metagenomics Sequencing. All segment samples from each participant were pooled and considered representative of the whole colon to minimize technical errors and variations. DNA samples were centrifuged at $5000 \times \mathrm{g}$ for 15 minutes, and the supernatants were discarded. The pellet was resuspended in $10 \mathrm{~mL}$ lysis buffer (0.5 M Tris-HCl; 20 mM EDTA; $10 \mathrm{mM} \mathrm{NaCl;} \mathrm{0.1 \%} \mathrm{SDS;}$ $\mathrm{pH}$ 9.0), and the mixtures were homogenized by centrifuging and shaking for 5-10 minutes.

Samples were then diluted $(1: 2)$ with a $10 \mathrm{~mL}$ lysis buffer and homogenized for another 5 minutes. Genomic DNA (gDNA) was precipitated by adding $5 \mathrm{~mL} 7.5 \mathrm{M}$ ammonium acetate and $25 \mathrm{~mL}$ ice-cold ethanol (95-100\%). The samples were subsequently incubated at $-20^{\circ} \mathrm{C}$ for $20-30$ minutes, and gDNA was collected following centrifugation at $4500 \times \mathrm{g}$ for 15 minutes at room temperature. DNA pellets were resuspended in $600 \mu \mathrm{L}$ TE buffer (Tris-EDTA, pH 8.0) and incubated at $65^{\circ} \mathrm{C}$ for 15 minutes. An equal volume of phenol-chloroform: isoamyl alcohol solution was briefly mixed with the DNA, and the mixtures were centrifuged at $12,000 \times \mathrm{g}$ for 5 minutes at room temperature. The supernatant aqueous phase was then transferred to a new tube, while 


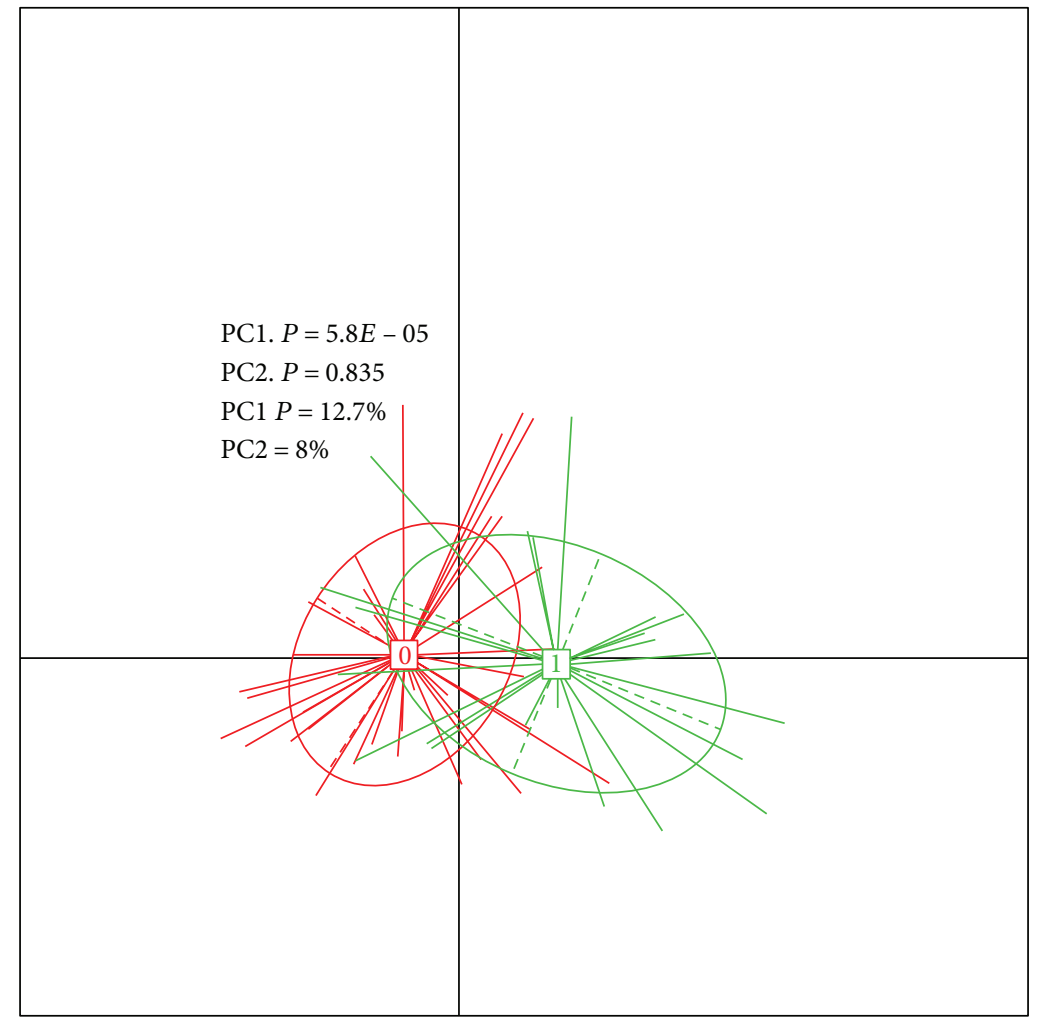

FIgure 1: Principal coordinate analysis (PCoA) between cases and controls.

the interface and the organic phase were discarded. This step was repeated until no protein was visible at the interface. The final supernatant aqueous phase was then transferred to a new tube; twice the amount of ethanol was added to the aqueous phase, and the solution was stored overnight. The following day, the DNA was ethanol precipitated as described above. The resulting DNA pellet was resuspended in $50 \mu \mathrm{L}$ TE buffer. The quality and concentration of the extracted gDNA were verified using 1\% agarose gel electrophoresis and a Qubit fluorometer 3.0 (Life Technologies, Carlsbad, CA).

2.6. Metagenomics DNA Library Construction and Sequencing. Paired-end (PE) metagenomics DNA library construction was performed, based on the manufacturer's instructions (Sequencing Kits and Reagents, Illumina, San Diego, CA). High-quality reads were separated from lowquality reads with " $\mathrm{N}$ " bases, adapter contamination, or human DNA contamination from the Illumina raw data using the BWA-SW algorithm (Li H, Durbin R. 2010, Bioinformatics). On average, the proportion of high-quality reads in all samples was approximately $98.1 \%$, and the insert sizes of our PE clones ranged from $313 \mathrm{bp}$ to $381 \mathrm{bp}$. Sequencing and data processing were performed at the Beijing Genomics Institute, where Illumina GAIIx and HiSeq 2000 platforms were utilized to sequence the samples.

2.7. Gene Catalog Construction. The first 72 randomly chosen samples were combined to establish the nonredundant gene set. Predicted open reading frames (ORFs) of the 72 samples were aligned to each other, and gene pairs with higher than 95\% identity were grouped. Groups with similar genes were merged, and the longest ORF in each group was used to represent that group. We therefore organized the nonredundant gene set from all predicted genes by excluding redundant ORFs. For 159 highquality reads in stages I, II, and III, we performed de novo assembly and gene predictions using SOAPdenovo v1.06 (Luo et al.: "SOAPdenovo2: An Empirically Improved Memory-Efficient Short-Read De Novo Assembler". GigaScience 20121 : 18) and GeneMark v2.7 (Ter-Hovhannisyan, Vardges, et al. "Gene Prediction in Novel Fungal Genomes Using an Ab Initio Algorithm with Unsupervised Training"; Genome Research 18.12 (2008): 1979-1990.), respectively. All predicted genes were aligned pair-wise using BLAT, and genes that could be aligned ( $>90 \%$ of gene length) to another gene with more than $95 \%$ identity (no gaps allowed) were removed as redundancies, resulting in a nonredundant gene catalog. This catalog of colonic samples was further combined with the previously constructed Meta HIT gene catalog by eliminating redundancies in the same manner.

2.8. Bioinformatics Analysis Pipeline. We subjected multiple samples from the mucosal microbiota metagenome to comparative phylogenetic analyses to understand the ecology of cultivation-independent gut microbiota and the phylogenetic differences between samples (Figure 1). We aligned all highquality reads to known bacterial, fungal, protozoal, or human gut gene databases from NCBI, RDP, or MetaHIT. For each 
TABle 1: Demographic data of study participants.

\begin{tabular}{lcr}
\hline & Age range & $38-77$ years \\
Demographic data & Gender ratio M:F & $15: 14$ \\
& BMI $>30$ & $18 / 29(62 \%)$ \\
\hline Symptoms at presentation & Rectal bleeding & $11 / 29(37 \%)$ \\
\hline & Anemia & $14(48 \%)$ \\
& Rectal growth & $2(6 \%)$ \\
Location of tumor & Descending colon growth & $4(13 \%)$ \\
& Transverse colon growth & $1(3 \%)$ \\
& Hepatic flexure growth & $8(27 \%)$ \\
\hline
\end{tabular}

sample, we compared paired alignment and single alignments to the databases.

2.9. Phylogenetic Classification of ORFs. Taxonomic assignments were performed with the BLASTp alignment tool against the NR90 database. Alignment hits with $E$ values greater than $1 \mathrm{E}-5$ were removed, and significant matches with $E$ values in the same order of the top hit were used for determining taxonomic groups. We assessed the taxonomic association of each gene by a lowest common ancestor(LCA-) based algorithm implemented in MEGAN43.

2.10. Statistical Analysis. The module extracted from the ipath (reference) test was assessed by means of the Wilcoxon test between the control and the cancer groups. The Chaol Richness Index, Shannon Index, and Simpson Diversity Index were used to describe the $\alpha$ diversity features of our bacterial community. A $P$ value of $<0.05$ was considered significant.

Beta diversity was used to assess diversity between cases and controls. Principal coordinate analysis (PCoA) based on the unweighted UniFrac distance metrics was used to demonstrate that there was a difference in the mucosal bacterial communities between the cases and controls, which was confirmed by permutational multivariate analysis of variance (PERMANOVA) PCoA in the CRC patients.

\section{Results}

Thirty-two patients were enrolled for the CRC metagenomics study to begin with, but of these, three were excluded because of low DNA quantity in their samples. Finally, the data on 29 confirmed patients with CRC were analyzed along with a group of age- and gender-matched controls. In this study, there were 14 females and 15 males aged from 38 to 77 years.

The majority 11 (37\%) of our patients were overweight $(\mathrm{BMI}=25-30)$. Five $(24 \%)$ patients were assigned to obesity class I $(\mathrm{BMI}=35-40)$. Obesity class III $\mathrm{BMI}>40$ was observed in a 54-year-old male subject. Only 4 (13\%) patients had normal BMI $(\mathrm{BMI}=18-25)$. One patient in our study was observed to be mildly thin (BMI $=17-18.5)$, a 66 -yearold female who had a BMI of just 15.66 as shown in Table 1.

Rectal bleeding was the commonest $(18 / 29,62 \%)$ presentation characteristic in the CRC group, and $11 / 29$ or $37 \%$ of patients presented with symptomatic anemia. On colonoscopic examination, the CRC location was rectal in 14 (48\%), and cecal growth was observed in 8 (27\%). There was also hepatic flexure growth in $1(3 \%)$ and descending colonic growth in $2(6 \%)$, and $4(13 \%)$ patients had transverse colon growth (Table 1). None of our patients had a familial colon cancer syndrome or any family history of colon cancer.

The histology of CRC was that of adenocarcinoma which was confirmed by two histopathologists experienced in GI histology in all patients.

The metagenomics analysis was carried out in cases and control as shown in Table 2, and in total, $3.58 \mathrm{G}$ reads were sequenced, with about $321.91 \mathrm{G}$ data being used in the analysis. The useful reads in each sample were aligned to $4.3 \mathrm{M}$ gene set 1 by soap 2 . On average, $70.33 \%$ reads can be mapped to the gene set; the max ration can reach $81.36 \%$.

The Chaol Richness Index, Shannon Index, and Simpson Diversity Index used to describe the $\alpha$ diversity features of our bacterial community are shown in Figures 2 and 3. The module extracted from the ipath (reference) test by the Wilcoxon test between the CRC and the control groups was 0.23 .

All the results were mapped to $9.9 \mathrm{M}$ gene sets 9 and 10 . The difference between the groups in rarefaction was quite significant, as shown in Figure 1. However, the Shannon alpha diversity showed no significant difference between the diseased group and the control group, as shown in Figure 3.

Beta diversity showed significant diversity between cases and controls. Principal coordinate analysis (PCoA) based on the unweighted UniFrac distance metrics demonstrated that there was a separation in the mucosal bacterial communities between the cases and controls, which was confirmed by permutational multivariate analysis of variance (PERMANOVA) PCoA in colorectal cancer, as shown in Figure 1.

It was further observed that the CRC group had statistically significantly higher 11 genera compared to those in the controls, as shown in Table 3. These genera were Atopobium, Beggiatoa, Burkholderia, Collinsella, Comamonas, Finegoldia, Fusobacterium, Gemella, Listeria, Methanobrevibacter, Parvimonas, Peptoniphilus, Peptostreptococcus, Porphyromonas, Selenomonas, Shuttleworthia, Solobacterium, Thermoanaerobacter, Verrucomicrobiales, and Yersinia. The enrichment of bacteria in colorectal cases is shown in Figure 4. 
TABLE 2: Metagenomic analysis in cases and controls.

\begin{tabular}{|c|c|c|c|c|}
\hline \multirow{2}{*}{ Genus, phylum, class, order, family } & \multirow{2}{*}{$P$ value } & \multicolumn{2}{|c|}{ Mean rank sum } & \multirow{2}{*}{$\mathrm{E}$} \\
\hline & & Controls & Cases & \\
\hline Abiotrophia, Firmicutes, Bacilli, Lactobacillales, Aerococcaceae & 0.0066 & 24 & 37 & 0 \\
\hline Acidaminococcus, Firmicutes, Negativicutes, Selenomonadales, Acidaminococcaceae & 0.0082 & 24 & 37 & 0 \\
\hline Akkermansia, Verrucomicrobia, Verrucomicrobiae, Verrucomicrobiales, Verrucomicrobiaceae & 0.0005 & 22 & 38 & 0 \\
\hline Alcaligenes, Proteobacteria, Betaproteobacteria, Burkholderiales, Alcaligenaceae & 0.0066 & 36 & 31 & 1 \\
\hline Anaerostipes, "Firmicutes," Clostridia, Clostridiales, Lachnospiraceae & 0.0005 & 22 & 38 & 0 \\
\hline Azoarcus, Proteobacteria, Betaproteobacteria, Rhodocyclales, Rhodocyclaceae & 0.0086 & 39 & 29 & 1 \\
\hline Bacteroides, Bacteroidetes, Bacteroidia, Bacteroidales, Bacteroidaceae & 0.0072 & 24 & 37 & 0 \\
\hline Beggiatoa, Proteobacteria, Gammaproteobacteria, Thiotrichales, Thiotrichaceae & 0.0003 & 44 & 26 & 1 \\
\hline Burkholderia, Proteobacteria, Betaproteobacteria, Burkholderiales, Burkholderiaceae & 0.0028 & 42 & 27 & 1 \\
\hline Butyrivibrio, Firmicutes, Clostridia, Clostridiales, Lachnospiraceae & 0.0005 & 22 & 38 & 0 \\
\hline Chlorobium, Chlorobi, Chlorobia, Chlorobiales, Chlorobiaceae & 0.0027 & 39 & 29 & 1 \\
\hline Clostridium, Firmicutes, Clostridia, Clostridiales, Clostridiaceae & 0.0002 & 21 & 39 & 0 \\
\hline Coprococcus, Firmicutes, Clostridia, Clostridiales, Lachnospiraceae & 0.0052 & 24 & 37 & 0 \\
\hline Coriobacterium, Actinobacteria, Actinobacteria, Coriobacteriales, Coriobacteriaceae & 0.0083 & 38 & 29 & 1 \\
\hline Coxiella, Proteobacteria, Gammaproteobacteria, Legionellales, Coxiellaceae & 0.0066 & 36 & 31 & 1 \\
\hline Crenothrix, Bacteroidetes, Sphingobacteria, Sphingobacteriales, Crenotrichaceae & 0.0013 & 42 & 27 & 1 \\
\hline Cryptobacterium, Actinobacteria, Actinobacteria, Coriobacteriales, Coriobacteriaceae & 0.0027 & 39 & 29 & 1 \\
\hline Dethiobacter, Firmicutes, Clostridia, Clostridiales, Syntrophomonadaceae & 0.0084 & 40 & 28 & 1 \\
\hline Enterobacter, Proteobacteria, Gammaproteobacteria, Enterobacteriales, Enterobacteriaceae & 0.0004 & 22 & 39 & 0 \\
\hline Eubacterium, Firmicutes, Clostridia, Clostridiale, Eubacteriaceae & 0.0026 & 23 & 38 & 0 \\
\hline Haemophilus, Proteobacteria, Gammaproteobacteria, Pasteurellales, Pasteurellaceae & 0.0060 & 24 & 37 & 0 \\
\hline Haladaptatus, Archaea, Euryarchaeota, Halobacteria, Halobacteriales, Haladaptatus & 0.0007 & 38 & 30 & 1 \\
\hline Holdemania, Firmicutes, Erysipelotrichi, Erysipelotrichales, Erysipelotrichidae & 0.0004 & 22 & 39 & 0 \\
\hline Klebsiella, Proteobacteria, Gammaproteobacteria, Enterobacteriales, Enterobacteriaceae & 0.0066 & 24 & 37 & 0 \\
\hline Listeria, Firmicutes, Bacilli, Bacillales, Listeriaceae & 0.0008 & 41 & 28 & 1 \\
\hline Megasphaera, Firmicutes, Negativicutes, Selenomonadales, Veillonellaceae & 0.0021 & 23 & 38 & 0 \\
\hline Mycoplasma, Tenericutes, Mollicutes, Mycoplasmatales, Mycoplasmataceae & 0.0025 & 40 & 28 & 1 \\
\hline Paracoccus, Proteobacteria, Alphaproteobacteria, Rhodobacterales, Rhodobacteraceae & 0.0075 & 41 & 28 & 1 \\
\hline Polaribacter, Bacteroidetes, Flavobacteria, Flavobacteriales, Flavobacteriaceae & 0.0002 & 43 & 27 & 1 \\
\hline Roseburia, Firmicutes, Clostridia, Clostridiales, Lachnospiraceae & 0.0057 & 24 & 37 & 0 \\
\hline Serratia, Proteobacteria, Gammaproteobacteria, Enterobacteriales, Enterobacteriaceae & 0.0002 & 41 & 28 & 1 \\
\hline Sphaerochaeta, Spirochaetes, Spirochaetia, Brachyspirales, Sarpulinacea & 0.0004 & 43 & 26 & 1 \\
\hline Sulfurovum, Proteobacteria, Epsilonproteobacteria & 0.0027 & 42 & 27 & 1 \\
\hline Ureaplasma, Tenericutes, Mollicutes, Mycoplasmatales, Mycoplasmataceae & 0.0034 & 39 & 29 & 1 \\
\hline Unclassified & 0.0031 & 23 & 38 & 0 \\
\hline
\end{tabular}

Further, the subanalysis of Enterobacteriaceae revealed that enterotype 1 was observed in 15 patients with CRC while enterotype 2 was found to be present in 2 cases. There were six patients with enterotype 3 positive among the CRC group. Enterotype 1 was observed to be more frequently present in the control subjects than in the CRC group (Figure 5).

\section{Discussion}

Carcinogenesis in colorectal cancer (CRC) represents a heterogeneous process with a differing set of somatic molecular alterations and can be influenced by a diet and environmental and microbial exposures. Recent evidence has shown a significant link between CRC and microbiota thus affirming the old ties of bacteria and colorectal carcinogenesis observed in the past. Various studies have indicated that the presence in the gut of Bacteroides vulgatus, Bacteroides stercoris, and Clostridia species have all been directly linked to a high risk of CRC [3]. It is hypothesized that some intestinal bacteria potentiate intestinal carcinogenesis by producing genotoxins, altering the immune response and intestinal microenvironment, and activating oncogenic signaling pathways [11].

In this study, we intentionally choose metagenomic studies on colonic washes rather than on fecal samples as the evidence in support of mucosal microbiota mapping is profound. The mucosal microbiota maintains a closer interaction with the intestinal epithelium than that of the 

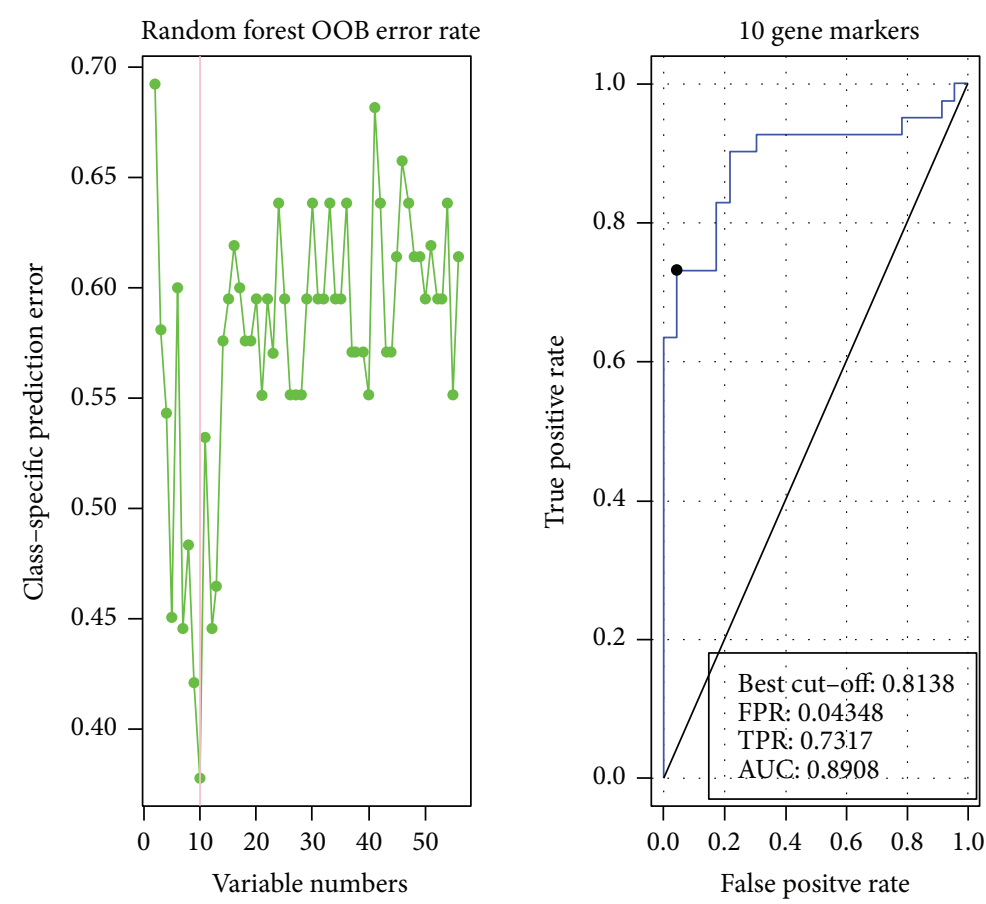

FIgURE 2: Classification based on mOTU marker in CRC cases and controls.

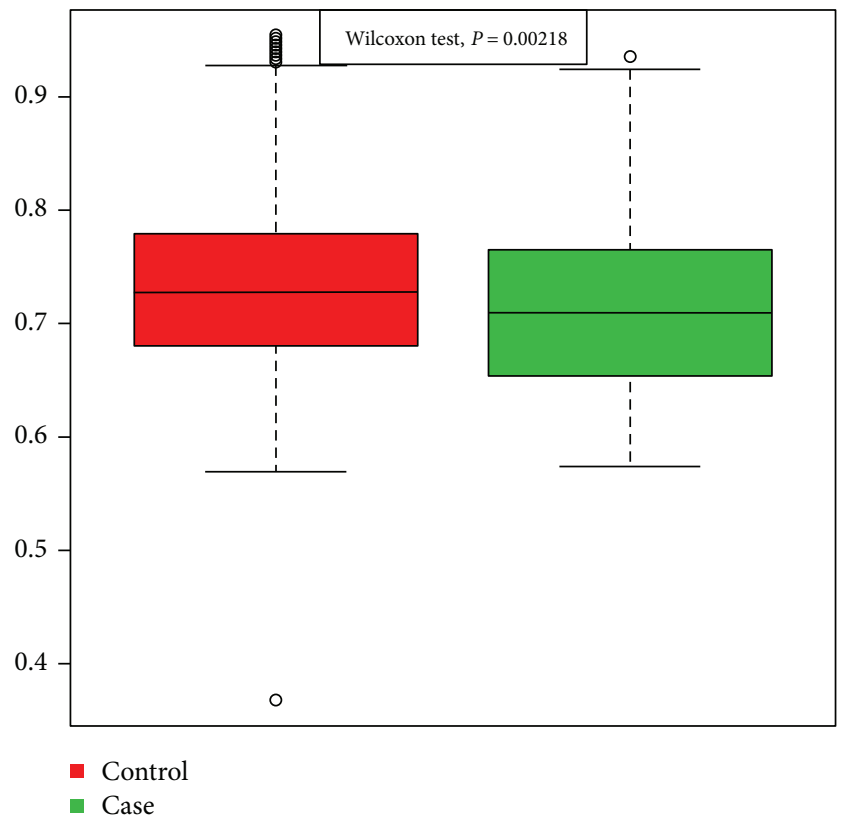

Figure 3: Alpha-Shannon Index in CRC cases and controls.

microbiota found in feces, and there is significant intersubject variability as well as differences between stool and mucosa community composition as has been demonstrated by Eckburg et al. [12].

The standard bowel prepration used in subjects in this study may be presumed to have altered the diversity of mucosa associated microbiota. Nevertheless, Harrell et al. observed that the taxonomic classification did not reveal significant changes at the phylum level, but only at the genus level. The authors of this study concluded that the degree of change underscores the importance of the need to consider the potentially influential effects of bowel preparation in experimental studies [13].

Our study showed that the CRC cases had significant enrichment of eleven genera compared to those in the control group, as shown in Table 3. The metagenomic sequencing showed that specific species, such as Fusobacterium nucleatum, Peptostreptococcus stomatis, and Parvimonas micra, were present in significantly greater quantities in the CRC patients than in the controls. Fusobacterium nucleatum has been identified to have a tumor-based immune evasion mechanism that is bacteria-dependent in the pathogenesis of CRC. Gur et al. have demonstrated that Fusobacterium nucleatum-bound tumors are protected from NK-mediated killing and immune cell attack due to an interaction between the fusobacterial protein Fap2 and the immune cell inhibitory receptor TIGIT on tumor-infiltrating lymphocytes including natural killer cells [14]. The multiplication of this genus in our population may possibly be a pointer to one of the target microbes for study in the future.

Another organism found to be quite significantly present in our study was $P$. anaerobius. It interacts with a toll-like receptor 2 (TLR2) and TLR4 on colon cells to increase the levels of reactive oxidative species, which promotes cholesterol synthesis and cell proliferation. Ni et al. have shown that the levels of $P$. anaerobius were found to be higher in human colon tumor tissues and adenomas when compared with nontumorous tissues. The authors of this study postulated that this bacterium increases colon dysplasia in a mouse model of CRC [15].

In animal studies, germ-free mice fed with stool from individuals with CRC developed significantly higher 
TABle 3: Significant enrichment in colorectal cases.

\begin{tabular}{lcc}
\hline Genus, phylum, class, order, family & $P$ value & Enrichment \\
\hline Atopobium, Actinobacteria, Coriobacteriia, Coriobacteriales, Coriobacteriaceae & 0.00047 & CRC \\
Beggiatoa, Proteobacteria, Gammaproteobacteria, Thiotrichales, Thiotrichaceae & 0.0002 & CRC \\
Burkholderia, Proteobacteria, Betaproteobacteria, Burkholderiales, Burkholderiaceae & 0.00011 & CRC \\
Collinsella, Actinobacteria, Actinobacteria, Coriobacteriales, Coriobacteriaceae & 0.00269 & CRC \\
Comamonas, Proteobacteria, Betaproteobacteria, Burkholderiales, Comamonadaceae & 0.00665 & CRC \\
Finegoldia, Firmicutes, Clostridia, Clostridiales, Peptoniphilaceae & 0.00726 & CRC \\
Fusobacterium, Firmicutes, Clostridia, Clostridiales, Peptoniphilaceae & 0.00751 & CRC \\
Gemella, Firmicutes, Bacilli, Bacillales & 0.00743 & CRC \\
Listeria, Firmicutes, Bacilli, Bacillales, Listeriaceae & 0.00657 & CRC \\
Methanobrevibacter, Euryarchaeota, Methanobacteria, Methanobacteriales, Methanobacteriaceae & $7.63 E-05$ & CRC \\
Peptostreptococcus, Firmicutes, Clostridia, Clostridiales, Peptostreptococcaceae & 0.00014 & CRC \\
Peptoniphilus, Firmicutes, Clostridia, Clostridiales & 0.00023 & CRC \\
Peptostreptococcus, Firmicutes, Clostridia, Clostridiales, Peptostreptococcaceae & 0.00142 & CRC \\
Porphyromonas, Bacteroidetes, Bacteroidetes, Bacteroidales, Porphyromonadaceae & 0.0066 & CRC \\
Selenomonas, Firmicutes, Negativicutes, Selenomonadales, Veillonellaceae & 0.00343 & CRC \\
Solobacterium, Firmicutes, Erysipelotrichi, Erysipelotrichales, Erysipelotrichidae & 0.00904 & CRC \\
Thermoanaerobacter, Firmicutes, Clostridia, Thermoanaerobacterales, Thermoanaerobacteraceae & 0.00043 & CRC \\
Verrucomicrobiales, Verrucomicrobia, Verrucomicrobiae, Verrucomicrobiales, Verrucomicrobiacea & $1.68 E-06$ & CRC \\
Yersinia, Proteobacteria, Gammaproteobacteria, Enterobacteriales, Yersiniacea & 0.0095 & CRC \\
\hline
\end{tabular}
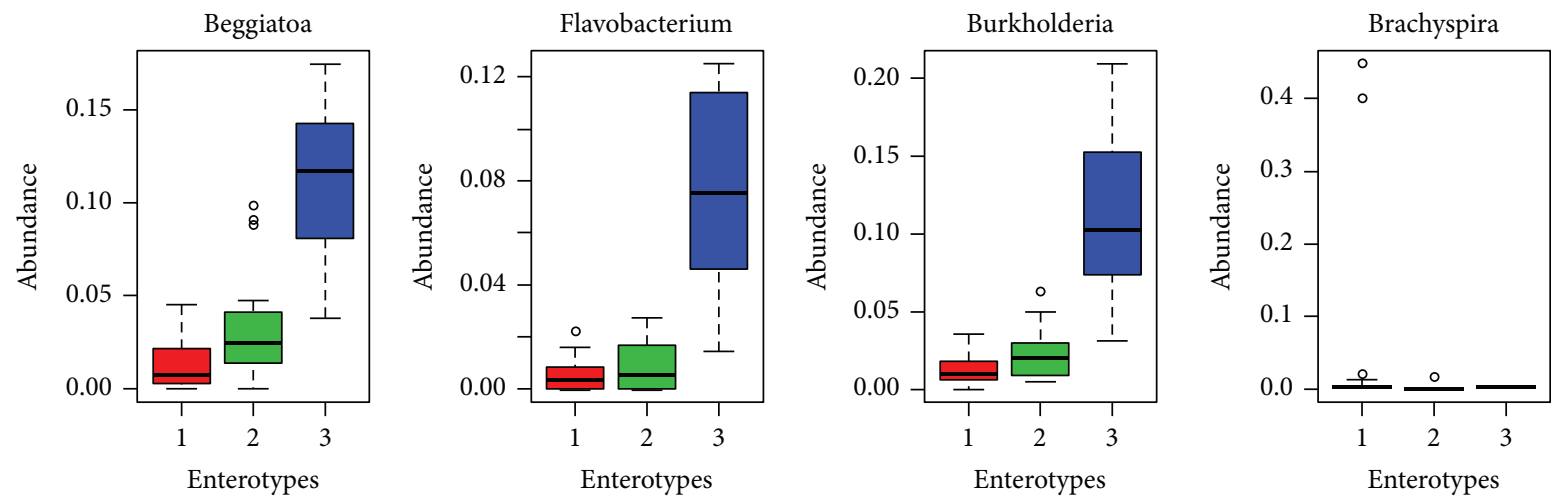

Figure 4: Principal coordinate analysis (PCA) in colorectal cancer patients.

proportions of high-grade dysplasia $(P<0.05)$ and macroscopic polyps $(P<0.01)$ than mice fed stools from controls [16]. This suggests that the fecal microbiota from patients with CRC can promote tumorigenesis in germ-free mice, connoting the CRC and microbiota relationship.

Fusobacterium, Selenomonas, and Peptostreptococcus were other genera which were present in significant quantities in our CRC patients. These butyrate-producing bacteria have been identified in colorectal cases by Hibberd et al. as well [17]. The presence of Firmicutes, Proteobacteria, and Bacteroidetes was again significantly higher in CRC patients as compared to controls, as shown in Table 3. In a study by $\mathrm{Xu}$ and Jiang [18], microbiota in the normal, cancer, and adenoma groups were observed. The authors found that bacteria with potential tumorigenesis, like Bacteroides fragilis and Fusobacterium, were more common in the carcinoma group, while some short-chain fatty acids (SCFA) producing microbes were more numerous in the healthy group. The commensal Escherichia were more abundant in the adenoma patients in their study. Authors describing the same research proposed that some bacteria, such as Butyricicoccus, E. coli, and Fusobacterium could possibly be used as potential biomarkers for the normal, adenoma, and cancer groups, respectively.

The majority of our study participants were obese. Obesity has been linked to colon cancer and also to diabetes. The role of microbiota in both of these conditions has been described [19]. It is possible that a sinister relationship between the two exists, and bearing in mind the global epidemics of obesity and diabetes, it may be prudent to mention that to mitigate the consequences of 


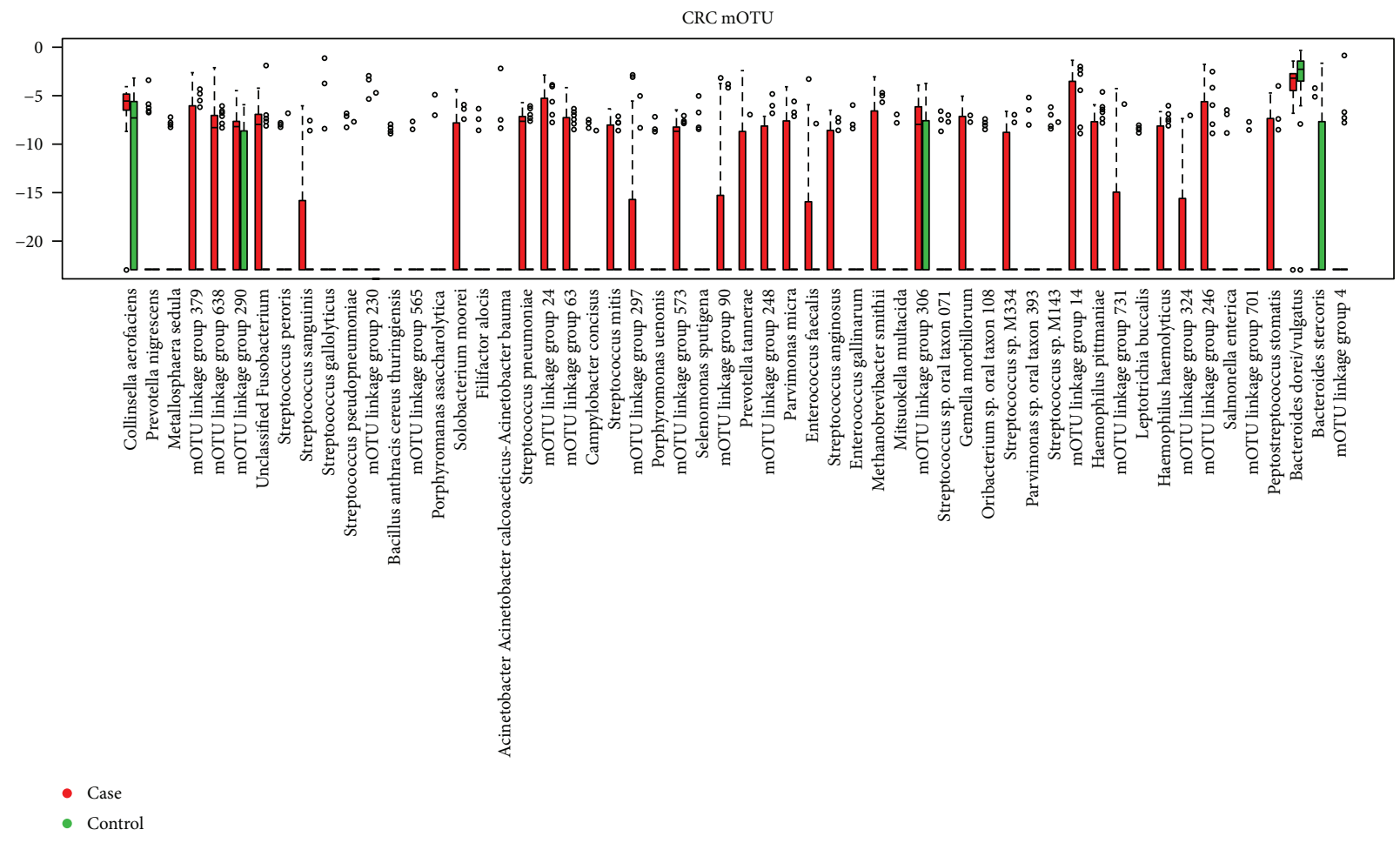

FIgURe 5: Control versus colorectal cancer case marker in mOTU.

colon cancer, both these modifiable factors should be addressed quite aggressively.

Genetic differences in intestinal microbiota in CRC patients were demonstrated by Goyal et al. [20], and the results of the current study identified changes in 11 genera in this sample of Saudi Arabian CRC patients. The limitation of our study is that the sample size was small but it nevertheless provides insights into the possible involvement of these gut microbes in CRC patients in this part of the globe.

The other side of the coin is whether knowledge of the potential role of microbiota in triggering CRC could suggest some protective interventions in colorectal carcinogenesis in the future. To address this issue, Hibberd et al. [17] observed how probiotic LGG exerted its beneficial effects and decreased the rate of CRC development. This probiotic intervention targeting microbiota could be used in humans in conjugation with other dietary supplements or drugs as part of prevention strategies for early-stage colon cancer, after further clinical validations.

To conclude, it is well known that CRC is one of the most treatable cancers, with a 5-year survival rate of approximately $64 \%$ [21]. These insights into the relationship between the microbiome, host genotype, and inflammation could suggest strategies for early diagnosis, preventive measures, and curative therapies for CRC. Further, it is anticipated that the study of microbiome dysbiosis may facilitate clinical application in CRC patient care. Hence, this study may be seen as a potential reference in this field when diagnostic tests for the early diagnosis of CRC, based on the analysis of gut microbiota, are finally discovered.

\section{Conflicts of Interest}

The authors declare that they have no conflicts of interest.

\section{Acknowledgments}

This research project was funded by King Abdulaziz City for Science and Technology (no. AT-29-243).

\section{References}

[1] S. Zou, L. Fang, and M. H. Lee, "Dysbiosis of gut microbiota in promoting the development of colorectal cancer," Gastroenterol Report, vol. 6, no. 1, pp. 1-12, 2018.

[2] Q. Zhu, R. Gao, W. Wu, and H. Qin, "The role of gut microbiota in the pathogenesis of colorectal cancer," Tumour Biology, vol. 34, no. 3, pp. 1285-1300, 2013.

[3] W. E. Moore and L. H. Moore, "Intestinal floras of populations that have a high risk of colon cancer," Applied and Environmental Microbiology, vol. 61, no. 9, pp. 3202-3207, 1995.

[4] M. N. Khabaz, J. A. Al-Maghrabi, T. Nedjadi et al., "Does Val/Val genotype of GSTP1 enzyme affects susceptibility to colorectal cancer in Saudi Arabia?," Neuro Endocrinology Letters, vol. 37, no. 1, pp. 46-52, 2016.

[5] R. N. Alharithy, "Polymorphisms in RETN gene and susceptibility to colon cancer in Saudi patients," Annals of Saudi Medicine, vol. 34, no. 4, pp. 334-339, 2014.

[6] S. A. Elsamany, A. S. Alzahrani, M. M. Mohamed et al., "Clinico-pathological patterns and survival outcome of colorectal cancer in young patients: western Saudi Arabia experience," Asian Pacific Journal of Cancer Prevention, vol. 15, no. 13, pp. 5239-5243, 2014. 
[7] M. A. Shalaby, H. A. Nounou, M. S. Alanazi, O. Alharby, N. Azzam, and H. M. Saeed, "Associations between single nucleotide polymorphisms of COX-2 and MMP-2 genes and colorectal cancer susceptibility in the Saudi population," Asian Pacific Journal of Cancer Prevention, vol. 15, no. 12, pp. 4989-4994, 2014.

[8] H. Alkhalidi and H. Kfoury, "Status of mismatch repair genes $h M S H 2$ and $h M S H 6$ in colorectal cancer in Saudi patients: an immunohistochemical analysis," Eastern Mediterranean Health Journal, vol. 18, no. 11, pp. 1114-1117, 2012.

[9] S. G. Daniel, C. L. Ball, D. G. Besselsen, T. Doetschman, and B. L. Hurwitz, "Functional changes in the gut microbiome contribute to transforming growth factor $\beta$-deficient colon cancer," mSystems, vol. 2, no. 5, pp. e00065-e00017, 2017.

[10] I. Allali, S. Delgado, P. I. Marron et al., "Gut microbiome compositional and functional differences between tumor and non-tumor adjacent tissues from cohorts from the US and Spain," Gut Microbes, vol. 6, no. 3, pp. 161-172, 2015.

[11] R. Shigefuku, T. Watanabe, Y. Kanno et al., "Fusobacterium nucleatum detected simultaneously in a pyogenic liver abscess and advanced sigmoid colon cancer," Anaerobe, vol. 48, pp. 144-146, 2017.

[12] P. B. Eckburg, E. M. Bik, C. N. Bernstein et al., "Diversity of the human intestinal microbial flora," Science, vol. 308, no. 5728, pp. 1635-1638, 2005.

[13] L. Harrell, Y. Wang, D. Antonopoulos et al., "Standard colonic lavage alters the natural state of mucosal-associated microbiota in the human colon," PLoS One, vol. 7, no. 2, article e32545, 2012.

[14] C. Gur, O. Mandelboim, and G. Bachrach, "“Messieurs, c'est les microbes qui auront le dernier mot": gentlemen, it is the microbes who have the last word (Louis Pasteur) - Fusobacterium nucleatum protect tumors from killing by immune cells," OncoImmunology, vol. 4, no. 9, article e1038690, 2015.

[15] Y. Ni, V. H. Y. Wong, W. C. S. Tai et al., “A metagenomic study of the preventive effect of Lactobacillus rhamnosus GG on intestinal polyp formation in Apc ${ }^{\mathrm{min} /+}$ mice," Journal of Applied Microbiology, vol. 122, no. 3, pp. 770-784, 2017.

[16] S. H. Wong, L. Zhao, X. Zhang et al., "Gavage of fecal samples from patients with colorectal cancer promotes intestinal carcinogenesis in germ-free and conventional mice," Gastroenterology, vol. 153, no. 6, pp. 1621-1633.e6, 2017.

[17] A. A. Hibberd, A. Lyra, A. C. Ouwehand et al., "Intestinal microbiota is altered in patients with colon cancer and modified by probiotic intervention," BMJ Open Gastroenterology, vol. 4, no. 1, article e000145, 2017.

[18] K. Xu and B. Jiang, "Analysis of mucosa-associated microbiota in colorectal cancer," Medical Science Monitor, vol. 23, pp. 4422-4430, 2017.

[19] H. Tilg, "Obesity, metabolic syndrome, and microbiota: multiple interactions," Journal of Clinical Gastroenterology, vol. 44, Supplement 1, pp. S16-S18, 2010.

[20] S. Goyal, P. Nangia-Makker, L. Farhana, Y. Yu, and A. P. N. Majumdar, "Racial disparity in colorectal cancer: gut microbiome and cancer stem cells," World Journal of Stem Cells, vol. 8, no. 9, pp. 279-287, 2016.

[21] J. M. Weiss, P. R. Pfau, E. S. O'Connor et al., "Mortality by stage for right- versus left-sided colon cancer: analysis of surveillance, epidemiology, and end results-Medicare data," Journal of Clinical Oncology, vol. 29, no. 33, pp. 4401-4409, 2011. 


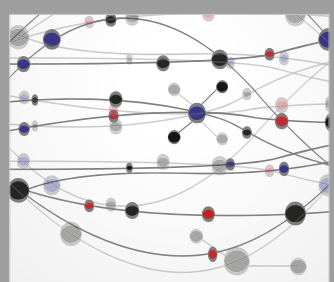

The Scientific World Journal
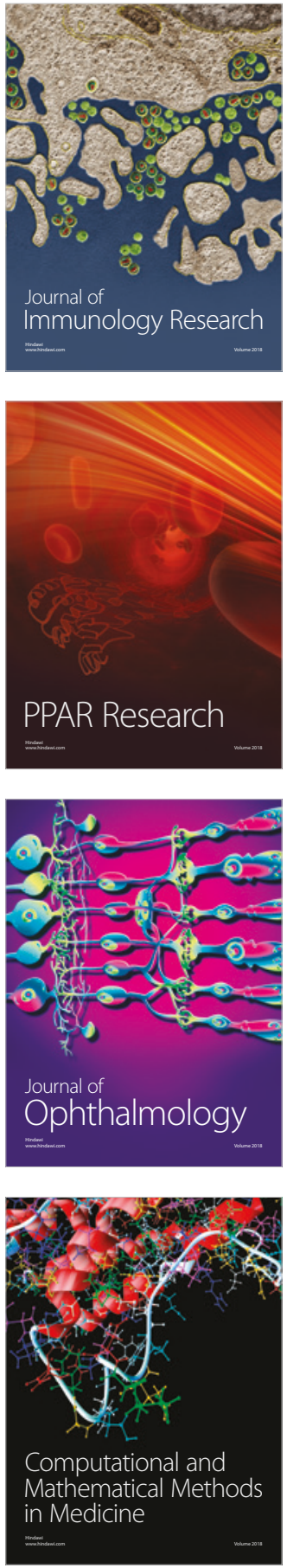

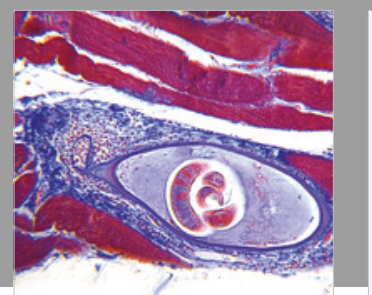

Gastroenterology Research and Practice

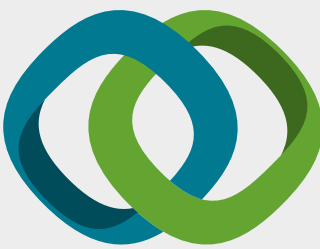

\section{Hindawi}

Submit your manuscripts at

www.hindawi.com
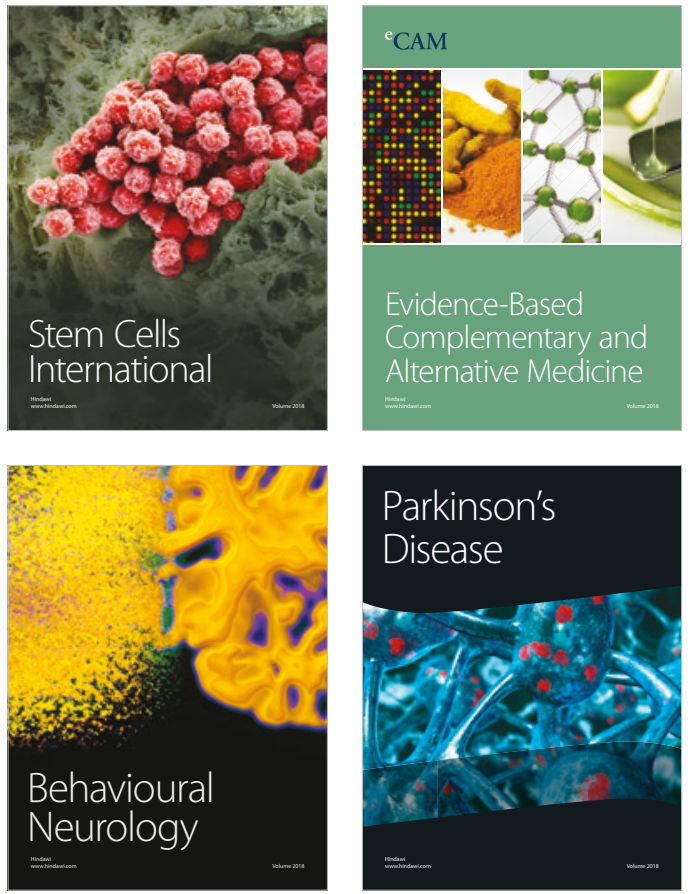

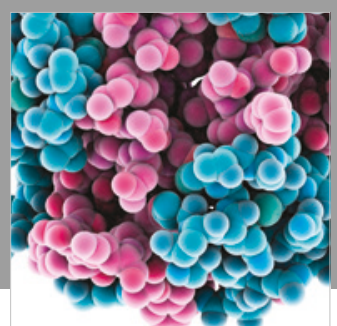

ournal of

Diabetes Research

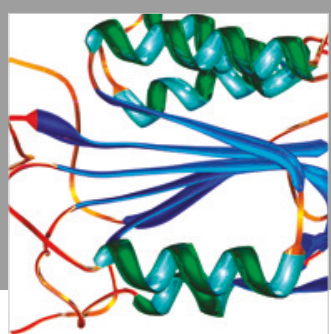

Disease Markers
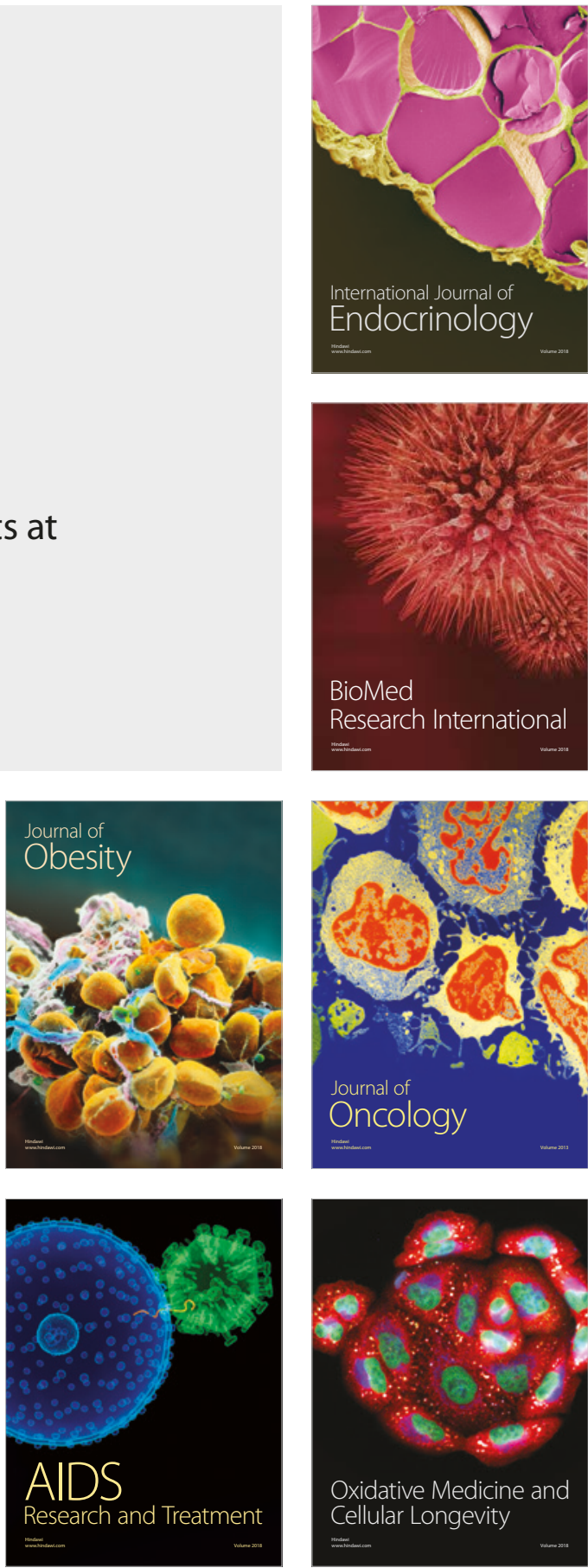